Article

\title{
Forecasting Frequent Alcohol Use among Adolescents in HBSC Countries: A Bayesian Framework for Making Predictions
}

\author{
Lorena Charrier $^{1}{ }^{(D)}$, Michela Bersia ${ }^{1,2}$, Alessio Vieno $^{3}\left(\mathbb{D}\right.$, Rosanna Irene Comoretto $^{1, *(D)}$,

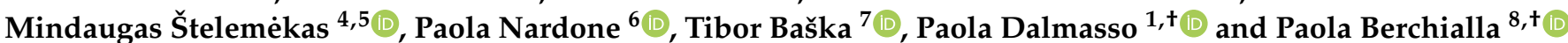

Citation: Charrier, L.; Bersia, M.; Vieno, A.; Comoretto, R.I.;

Štelemèkas, M.; Nardone, P.; Baška,

T.; Dalmasso, P.; Berchialla, P.

Forecasting Frequent Alcohol Use among Adolescents in HBSC

Countries: A Bayesian Framework

for Making Predictions. Int. J.

Environ. Res. Public Health 2022, 19,

2737. https://doi.org/10.3390/

ijerph19052737

Academic Editor: Paul B. Tchounwou

Received: 31 January 2022

Accepted: 24 February 2022

Published: 26 February 2022

Publisher's Note: MDPI stays neutral with regard to jurisdictional claims in published maps and institutional affiliations.

Copyright: (C) 2022 by the authors. Licensee MDPI, Basel, Switzerland. This article is an open access article distributed under the terms and conditions of the Creative Commons Attribution (CC BY) license (https:// creativecommons.org/licenses/by/ $4.0 /)$
1 Department of Public Health and Pediatrics, University of Torino, 10126 Torino, Italy; lorena.charrier@unito.it (L.C.); michela.bersia@edu.unito.it (M.B.); paola.dalmasso@unito.it (P.D.)

2 Post Graduate School of Medical Statistics, University of Torino, 10126 Torino, Italy

3 Department of Developmental and Social Psychology, University of Padova, 35131 Padova, Italy; alessio.vieno@unipd.it

4 Health Research Institute, Faculty of Public Health, Lithuanian University of Health Sciences, 47181 Kaunas, Lithuania; mindaugas.stelemekas@1smuni.lt

5 Department of Preventive Medicine, Faculty of Public Health, Lithuanian University of Health Sciences, 47181 Kaunas, Lithuania

6 National Centre for Disease Prevention and Health Promotion, Italian National Institute of Health, 00161 Rome, Italy; paola.nardone@iss.it

7 Department of Public Health, Jessenius Faculty of Medicine in Martin, Comenius University in Bratislava, 03601 Martin, Slovakia; tibor.baska@uniba.sk

8 Department of Clinical and Biological Sciences, University of Torino, 10043 Orbassano, Italy; paola.berchialla@unito.it

* Correspondence: rosannairene.comoretto@unito.it; Tel.: +39-011-670-6322

+ These authors equally contributed to this work.

Abstract: (1) Aim: To summarize alcohol trends in the last 30 years (1985/6-2017/8) among 15-year-olds in Health Behaviour in School-aged Children (HBSC) countries (overall sample size: 413,399 adolescents; $51.55 \%$ girls) and to forecast the potential evolution in the upcoming 2021/22 HBSC survey. (2) Methods: Using 1986-2018 prevalence data on weekly alcohol consumption among 15-year-olds related to 40 HBSC countries/regions, a Bayesian semi-parametric hierarchical model was adopted to estimate trends making a clusterization of the countries, and to give estimates for the 2022 HBSC survey. (3) Results: An overall declining trend in alcohol consumption was observed over time in almost all the countries. However, compared to 2014, some countries showed a new increase in 2018 and $2021 / 22$ estimates forecast a slight increase in the majority of countries, pointing out a potential bounce after a decreasing period in frequent drinking habits. (4) Conclusions: The clusterization suggested a homogenization of consumption habits among HBSC countries. The comparison between 2022 observed and expected data could be helpful to investigate the effect of risk behaviour determinants, including the pandemic impact, occurring between the last two waves of the survey.

Keywords: adolescents; alcohol; drinking; risk behaviour; trend; forecast; Bayesian longitudinal model

\section{Introduction}

Excessive alcohol use has been identified as one of the major preventable risk factors for population health globally having a significant impact on many health-related indicators, as pointed out by the United Nations (UN) Sustainable Development Goals (SDGs) target for "Strengthen the prevention and treatment of substance abuse, including narcotic drug abuse and harmful use of alcohol" [1]. The WHO's Non-Communicable Disease (NCD) Global Monitoring Framework sets a voluntary target to reduce the harmful use of alcohol as one of the NCD reduction aims till year 2025 [2].

The high public health burden of alcohol consumption is particularly critical in the young age group (15-19 years old), where alcohol attributable mortality accounts for around 
one-fifth of all deaths [3]. In addition to fatal consequences, adolescent alcohol use is often associated with other risky behaviours, such as other substance use and unprotected sex; the literature confirms the co-occurrence of multiple health risk behaviours and the idea that they tend to cluster together [4,5]. Negative psychological, social and physical health effects (school failure, violence, injuries, suicide attempts, sexually transmitted diseases and unintended pregnancy, among others) have also been observed. Moreover, the earlier the drinking initiation, the more severe the consequences, with an increased risk of alcohol abuse and a greater likelihood of substance use disorders in later life [6].

Adolescent alcohol use in Europe has shown a declining tendency in recent decades $[7,8]$ even if it has not followed homogeneous patterns among countries. This trend initially was not noticeable in Central and Eastern Europe and consumption even increased in some countries between 1998 and 2006 [9,10]. However, since 2006, the decline became apparent in other parts of the continent [11-13]. The decline was more pronounced in boys leading to a reduction in their traditional predominance above girls. Because of the mentioned changes over decades, heterogeneity across Europe has become less noticeable, and countries now show a more uniform picture. Such globalizing development of alcohol use among adolescents [14,15] can be caused by the gradual predominance of global factors over local ones. In fact, similar trends have been observed outside Europe, in Australia, New Zealand, Canada, Japan and USA [13].

Despite the recent declines, adolescent frequent alcohol consumption remains a major public health concern. In fact, because of its availability, alcohol is still one of the most used drugs among young people [6]. Monitoring trends is thereby crucial to early detect changes over time and promptly implement tailored health promotion interventions. Moreover, the above-indicated global targets are closely associated with specific indicators such as alcohol per capita consumption or heavy episodic drinking among adults and adolescents. The Health Behaviour in School-aged Children (HBSC) study serves as an important source for several indicators to measure alcohol-related challenges relevant to both country-specific and regional assessment of health-related behaviours among adolescents (www.hbsc.org, accessed on 14 January 2022).

This study aims to summarize the HBSC alcohol-related data of the last three decades and forecast the trends in frequent alcohol consumption among 15-year-olds in the upcoming HBSC survey sample as a contribution to the monitoring process of this public health issue.

\section{Materials and Methods}

\subsection{Participants}

The HBSC surveillance is a World Health Organization (WHO) collaborative crossnational study that monitors adolescent health and well-being every 4 years in more than 50 European and North American countries and regions. It is a school-based survey that uses a self-report questionnaire administered to whole school classes. Data from each country are collected according to the HBSC internationally approved protocol for each survey round to ensure consistency in survey instruments (such as a list of mandatory questions), data collection and processing procedures [16,17]. Samples are designed to be nationally representative of pupils aged 11, 13 and 15 years. Data for this study are based on 15-year-old adolescents, the age group with higher alcohol consumption prevalence [6].

For our analyses, we used HBSC data collected by the 40 countries that took part in at least three out of nine survey waves from 1985/6 and that had comparable data on alcohol consumption collected through the same question, mandatory until 2013/14, for at least one of the last two surveys (2013/14 and 2017/18). The countries/regions that met the inclusion criteria (see also Table A1 in Appendix A) were: Armenia (AM), Austria (AT), Flemish Belgium (BE-VLG), French Belgium (BE-WAL), Bulgaria (BG), Canada (CA), Switzerland $(\mathrm{CH})$, Czechia (CZ), Germany (DE), Denmark (DK), Estonia (EE), Spain (ES), Finland (FI), France (FR), England (GB-ENG), Scotland (GB-SCT), Wales (GB-WLS), Greenland (GL), Greece (GR), Croatia (HR), Hungary (HU), Ireland (IE), Israel (IL), Iceland (IS), Italy (IT), 
Lithuania (LT), Luxembourg (LU), Latvia (LV), former Yugoslav Republic of Macedonia (MK), Malta (MT), Netherland (NL), Norway (NO), Poland (PL), Portugal (PT), Romania (RO), Russian Federation (RU), Sweden (SE), Slovenia (SI), Slovakia (SK), and Ukraine (UA).

Analyses were stratified by gender due to the differences in both prevalence and trend of frequent alcohol consumption between boys and girls.

\subsection{Measures}

Weekly/Daily Alcohol Use

Weekly/daily alcohol use was evaluated with the question 'At present, how often do you drink anything alcoholic? Try to include even those times when you only drink a small amount'. The items were beer, wine, spirits, alcopops, aperitifs, cider, cocktails and other when asked. For each item, response options were ' 1 = every day', '2 = every week', ' $3=$ every month' ' ' $4=$ rarely' and ' $5=$ never'. An overall alcohol use index was created considering the highest frequency of any alcoholic beverage consumed. The variable was dichotomized into 'at least weekly alcohol use' (response options 1 and 2) and 'less than weekly' (response options 3, 4 and 5).

The variable construction took into account the changes over time in the items included in the question mentioned above, due to changes in the consumption of specific alcoholic drinks: wine, beer and spirits have been present since the beginning of HBSC survey, cider was included only until the 1993/94 wave, while alcopops and 'other' have been added since the 2005/06 survey.

In $2017 / 18$, due to the modification of some mandatory questions, data on frequent alcohol consumption comparable to previous waves were available in only 23 of the 40 countries/regions included in this study: Armenia (AM), Austria (AT), Flemish Belgium (BE-VLG), French Belgium (BE-WAL), Bulgaria (BG), Switzerland (CH), Czechia (CZ), Denmark (DK), Estonia (EE), Scotland (GB-SCT), Wales (GB-WLS), Croatia (HR), Ireland (IE), Israel (IL), Italy (IT), Lithuania (LT), Latvia (LV), Malta (MT), Norway (NO), Russian Federation (RU), Sweden (SE), Slovenia (SI), and Slovakia (SK).

\subsection{Statistical Analysis}

A Bayesian semi-parametric hierarchical model was adopted to estimate the alcohol weekly consumption trend in each of the 40 included countries. Following the model introduced by Paddock et al. 2013 [18] for the analysis of longitudinal data,

$$
y_{i j}=\alpha+f\left(t_{i j}\right)+g_{i}\left(t_{i j}\right)+\epsilon_{i j}
$$

where $y_{i j}$ represents the alcohol weekly consumption observed for country $i$ at wave $j=\left(1, \ldots, m_{i}\right)$ and $m_{i}$ is the number of waves the $\mathrm{i}$-th country participated in and varies country-by-country. The term $f\left(t_{i j}\right)$ is a polynomial function of time to allow for non-linear trends and it takes the form $\left(t, t_{i j}, t_{i j}{ }^{2}, \ldots, t_{i j}{ }^{n}\right) \beta$, with $\beta$ being the vector of the fixed effect coefficients. Analogously, $g_{i}\left(t_{i j}\right)$ takes the form $\left(t, t_{i j}, t_{i j}{ }^{2}, \ldots, t_{i j}{ }^{n}\right) b_{i}$, with $b_{i}$ being the set of the random effects (country-level) coefficients.

To be as flexible as possible in modelling the trend, we adopted the Dirichlet Process (DP) framework introduced by Escobar and West [19] that generalizes the prior construction on random effects $b_{i}$ to non-parametric formulation, allowing estimation of a different form than a specified parametric distribution, typically Gaussian distribution.

Thus, the random effects are assumed to be generated from an unknown distribution $F$ and are assumed to be conditionally independent, given $F$

$$
b_{1}, \ldots b_{n} \sim F \text {, with } F \mid c, F_{o} \sim D P\left(c, F_{0}\right)
$$

$F_{0}$ represents the 'best guess' about the form of $\mathrm{F}$ prior to observing data and the precision parameter, $c>0$, expresses the degree of confidence that $F_{0}$ is the correct generating distribution for random effects, i.e., the higher the value of $\mathrm{c}$, the more $F$ is expected to conform to $F_{0} . F_{0}$ was chosen as normal distribution $N\left(0, \tau^{-1}\right)$. Flat priors for fixed effects 
$\beta$ and the global intercept $\alpha$ were considered. Independent $\operatorname{Gamma}(0.1,0.1)$ are defined for the precision parameters $\tau$.

The DP has the property to have a positive probability for ties to occur among the values of the random effects $b_{i}$. The set of unique values among the random effects are called clusters, and we exploited such properties of DP to borrow strength in trend estimation from countries who share the same random effects. Specifically, countries with similar trends will have higher probabilities of co-clustering.

We impose $c \sim \operatorname{Gamma}(a, 1)$, which encodes the prior expected number of clusters (the higher the values for shape parameter $a$ the higher the number of clusters).

To obtain the posterior estimates of the model parameters, we ran 100,000 MCMC iterations, with the first 30,000 as burn-in.

All the analyses were performed using $R$ version 4.1.2 [20].

\section{Results}

The overall sample size was 413,399 adolescents from the 40 countries that met the inclusion criteria; the mean age was 15.5 years, and $51.55 \%$ were girls.

\subsection{Prevalences of Weekly/Daily Alcohol Consumption from 1986 to 2018}

Tables 1 and 2 show the prevalence of weekly/daily alcohol consumption for each country and wave for boys and girls, respectively.

Table 1. Prevalence (\%) of weekly/daily alcohol consumption by country and wave from 1985/86 to $2017 / 18$ among boys.

\begin{tabular}{|c|c|c|c|c|c|c|c|c|c|}
\hline Country $^{1}$ & $1985 / 86$ & $1989 / 90$ & 1993/94 & $1997 / 98$ & $2001 / 02$ & $2005 / 06$ & $2009 / 10$ & $2013 / 14$ & $2017 / 18$ \\
\hline $\mathrm{AM}$ & & & & & & & 28.73 & 18.97 & 22.89 \\
\hline AT & & 37.83 & 47.3 & 39.57 & 28.82 & 41.25 & 37.3 & 22.85 & 23.86 \\
\hline BE-VLG & & & 44.82 & 38.93 & 37.87 & 41.67 & 31.63 & 17.60 & 18.46 \\
\hline BE-WAL & 54.01 & 40.95 & 45.87 & 45.95 & 30.91 & 37.85 & 26.4 & 17.02 & 16.17 \\
\hline BG & & & & & & 45.42 & & 32.43 & 49.70 \\
\hline CA & & 34.49 & 24.98 & 22.37 & 27.2 & 19.12 & 16.14 & 12.78 & \\
\hline $\mathrm{CH}$ & 22.24 & & & 19.64 & 33.42 & 26.98 & 26.51 & 11.36 & 11.17 \\
\hline $\mathrm{CZ}$ & & & 38.73 & 33.74 & 40.58 & 38.7 & 44.01 & 20.38 & 18.93 \\
\hline $\mathrm{DE}$ & & & 29.45 & 29.78 & 37.05 & 25.1 & 25.24 & 17.93 & \\
\hline DK & & & 41.82 & 48.13 & 50.39 & 38.61 & 26.75 & 20.39 & 25.27 \\
\hline $\mathrm{EE}$ & & & 14.71 & 23.48 & 28.11 & 51.01 & 29.04 & 16.95 & 11.34 \\
\hline ES & & & 25.78 & & 24.23 & & 22.97 & 10.79 & \\
\hline FI & 10.59 & 12.28 & 17.13 & 12.43 & 12.68 & 11.54 & 7.06 & 7.68 & \\
\hline FR & & & 40.1 & 33.58 & 19.95 & 24.44 & 28.25 & 18.25 & \\
\hline GB-ENG & & & & 18.58 & 51.03 & 39.14 & 24.79 & 10.47 & \\
\hline GB-SCT & 38.43 & 36.28 & 41.94 & 37.45 & 40.91 & 38.26 & 26.27 & 14.14 & 13.22 \\
\hline GB-WLS & 51.57 & 51.92 & 52.94 & 54.48 & 47.98 & 45.9 & 31.48 & 13.45 & 13.95 \\
\hline GL & & & 11.38 & 18.89 & 22.68 & 11.23 & 6.47 & 11.26 & \\
\hline GR & & & & 52.09 & 39.94 & 42.74 & 43.1 & 28.59 & \\
\hline HR & & & & & 34.31 & 44.63 & 43.27 & 34.26 & 29.22 \\
\hline $\mathrm{HU}$ & 24.48 & 25.06 & 23.93 & 33.23 & 42.31 & 34.68 & 31.01 & 29.06 & \\
\hline IE & & & & 29.26 & 14.97 & 17.96 & 13.6 & 5.11 & 6.52 \\
\hline IL & & & 24.23 & 24.53 & 23.96 & 21.61 & 28.74 & 29.22 & 17.70 \\
\hline IS & & & & & & 15.08 & 7.87 & 3.36 & \\
\hline IT & & & & & 46.83 & 47.68 & 39.33 & 31.55 & 30.31 \\
\hline LT & & & 15.67 & 18.12 & 27.63 & 25.47 & 24.41 & 13.65 & 9.01 \\
\hline LU & & & & & & 29.23 & 23.44 & 12.58 & \\
\hline LV & & & 18.64 & 29.41 & 19.78 & 31.03 & 26.68 & 9.46 & 6.77 \\
\hline MK & & & & & 21.56 & 28.47 & 23.35 & 18.78 & \\
\hline MT & & & & & 55.97 & 50 & & 31.85 & 26.13 \\
\hline NL & & & & & 44.53 & 42.29 & 27.03 & 18.01 & \\
\hline NO & & 8.47 & 9.95 & 17.05 & 16.71 & 21.41 & 10.99 & 4.06 & 6.85 \\
\hline PL & & 10.13 & 22.51 & 20.68 & 19.19 & 18.63 & 17.31 & 12.77 & \\
\hline $\mathrm{PT}$ & & & & 31.02 & 19.3 & 20.85 & 12.4 & 11.27 & \\
\hline RO & & & & & & 33.07 & 34.58 & 28.57 & \\
\hline RU & & & 21.39 & 27.61 & 31.83 & 42.4 & 12.85 & 12.16 & 6.03 \\
\hline SE & 14.34 & 27.55 & 21.76 & 21.29 & 18.73 & 15.35 & 10.63 & 5.08 & 7.53 \\
\hline SI & & & & & 33.02 & 58.58 & 54.43 & 25.14 & 20.11 \\
\hline SK & & & 33.78 & 33.33 & & 41.97 & 28.19 & 19.73 & 20.35 \\
\hline UA & & & & & 43 & 59.24 & 45.44 & 17.45 & \\
\hline
\end{tabular}

${ }^{1}$ AM (Armenia), AT (Austria), BE-VLG (Flemish Belgium), BE-WAL (French Belgium), BG (Bulgaria), CA (Canada), CH (Switzerland), CZ (Czechia), DE (Germany), DK (Denmark), EE (Estonia), ES (Spain), FI (Finland), FR (France), GB-ENG (England), GB-SCT (Scotland), GB-WLS (Wales), GL (Greenland), GR (Greece), HR (Croatia), HU (Hungary), IE (Ireland), IL (Israel), IS (Iceland), IT (Italy), LT (Lithuania), LU (Luxembourg), LV (Latvia), MK (former Yugoslav Republic of Macedonia.), MT (Malta), NL (Netherland), NO (Norway), PL (Poland), PT (Portugal), RO (Romania), RU (Russian Federation), SE (Sweden), SI (Slovenia), SK (Slovakia), UA (Ukraine). 
Table 2. Prevalence (\%) of weekly/daily alcohol consumption by country and wave from 1985/86 to 2017/18 among girls.

\begin{tabular}{|c|c|c|c|c|c|c|c|c|c|}
\hline Country $^{1}$ & $1985 / 86$ & $1989 / 90$ & $1993 / 94$ & $1997 / 98$ & $2001 / 02$ & $2005 / 06$ & $2009 / 10$ & $2013 / 14$ & $2017 / 18$ \\
\hline AM & & & & & & & 11.61 & 6.68 & 5.48 \\
\hline AT & & 18.64 & 29.09 & 23.96 & 22.59 & 34.94 & 28.29 & 11.25 & 18.42 \\
\hline BE-VLG & & & 25.86 & 22.31 & 24.44 & 24.12 & 15.79 & 10.74 & 7.94 \\
\hline BE-WAL & 36.73 & 26.93 & 28.77 & 28.24 & 20.11 & 20.78 & 19.1 & 9.58 & 8.45 \\
\hline BG & & & & & & 33.37 & & 17.65 & 35.09 \\
\hline CA & & 25.16 & 19.39 & 17.29 & 18.84 & 15.62 & 13.77 & 8.20 & \\
\hline $\mathrm{CH}$ & 9.86 & & & 9.18 & 14.65 & 15.93 & 13.08 & 6.32 & 3.81 \\
\hline $\mathrm{CZ}$ & & & 18.61 & 19.93 & 29.61 & 26.59 & 33.51 & 13.89 & 11.86 \\
\hline $\mathrm{DE}$ & & & 20.35 & 22.72 & 24.27 & 15.21 & 13.83 & 7.42 & \\
\hline DK & & & 35.01 & 38.56 & 39.91 & 28.3 & 17.72 & 12.52 & 17.81 \\
\hline $\mathrm{EE}$ & & & 5.18 & 11.48 & 12.04 & 41.16 & 24.56 & 11.89 & 6.31 \\
\hline ES & & & 24.52 & & 18.36 & & 22.78 & 8.21 & \\
\hline FI & 9.76 & 6.95 & 9.58 & 8.56 & 8.45 & 7.17 & 6.81 & 3.33 & \\
\hline FR & & & 17.82 & 16.01 & 10.47 & 13.31 & 15.38 & 8.95 & \\
\hline GB-ENG & & & & 27.75 & 43.72 & 37.82 & 17.6 & 9.20 & \\
\hline GB-SCT & 27.34 & 29.17 & 33.43 & 33.81 & 41.49 & 36.54 & 24.75 & 11.90 & 12.32 \\
\hline GB-WLS & 40.95 & 41.59 & 46.17 & 36.9 & 31.45 & 42.61 & 28.12 & 10.97 & 12.42 \\
\hline GL & & & 10.61 & 15.1 & 7.14 & 13.27 & 8.47 & 2.58 & \\
\hline GR & & & & 31 & 21.64 & 25.79 & 34.47 & 17.61 & \\
\hline $\mathrm{HR}$ & & & & & 20.79 & 29.75 & 26.68 & 13.86 & 14.31 \\
\hline $\mathrm{HU}$ & 9.73 & 5.62 & 14.57 & 11.63 & 20.75 & 23.25 & 19.76 & 18.23 & \\
\hline $\mathrm{IE}$ & & & & 12.37 & 11.78 & 18.78 & 9.7 & 3.51 & 5.03 \\
\hline IL & & & 11.42 & 8.56 & 11.49 & 9.77 & 9.63 & 9.76 & 6.60 \\
\hline IS & & & & & & 10.93 & 4.62 & 2.09 & \\
\hline IT & & & & & 29.48 & 30.58 & 26.36 & 17.02 & 17.61 \\
\hline LT & & & 6.76 & 10.5 & 13.01 & 20.04 & 16.81 & 6.01 & 7.12 \\
\hline LU & & & & & & 17.75 & 14.51 & 7.78 & \\
\hline LV & & & 5.56 & 12.57 & 12.62 & 25.07 & 21.08 & 4.02 & 5.43 \\
\hline MK & & & & & 7.28 & 14.76 & 12.64 & 9.09 & \\
\hline MT & & & & & 42.51 & 39.02 & & 26.48 & 18.70 \\
\hline NL & & & & & 24.47 & 28.51 & 17.21 & 12.89 & \\
\hline $\mathrm{NO}$ & & 8.03 & 7.08 & 13.46 & 17.19 & 21.19 & 9.57 & 3.56 & 2.74 \\
\hline PL & & 2.95 & 9.56 & 8.09 & 7.83 & 12.87 & 10.71 & 9.27 & \\
\hline PT & & & & 8.97 & 13.45 & 9.57 & 6.42 & 4.24 & \\
\hline $\mathrm{RO}$ & & & & & & 10.7 & 8.37 & 9.38 & \\
\hline RU & & & 10.51 & 24.51 & 18.43 & 36.12 & 9.29 & 5.64 & 2.07 \\
\hline SE & 11.61 & 18.16 & 12.4 & 13.53 & 11.89 & 11.13 & 7.58 & 3.08 & 5.15 \\
\hline SI & & & & & 19.92 & 33.61 & 41.78 & 18.27 & 14.17 \\
\hline SK & & & 10.83 & 17.13 & & 27.29 & 15.94 & 11.19 & 10.39 \\
\hline UA & & & & & 20.49 & 46.47 & 29.93 & 10.16 & \\
\hline
\end{tabular}

1 AM (Armenia), AT (Austria), BE-VLG (Flemish Belgium), BE-WAL (French Belgium), BG (Bulgaria), CA (Canada), CH (Switzerland), CZ (Czechia), DE (Germany), DK (Denmark), EE (Estonia), ES (Spain), FI (Finland), FR (France), GB-ENG (England), GB-SCT (Scotland), GB-WLS (Wales), GL (Greenland), GR (Greece), HR (Croatia), HU (Hungary), IE (Ireland), IL (Israel), IS (Iceland), IT (Italy), LT (Lithuania), LU (Luxembourg), LV (Latvia), MK (former Yugoslav Republic of Macedonia.), MT (Malta), NL (Netherland), NO (Norway), PL (Poland), PT (Portugal), RO (Romania), RU (Russian Federation), SE (Sweden), SI (Slovenia), SK (Slovakia), UA (Ukraine).

The data show an overall declining trend in frequent alcohol consumption over the last three decades, in almost all countries, both in boys and girls. For boys, the estimated trends showed an increase in alcohol consumption in the decade 1990-2000 followed by a decrease. A notable exception is Bulgaria (BG), for which, however, only the data of three survey waves $(2000,2014$ and 2018) were available. The same pattern is observed among girls. However, Canada (CA) is a notable exception, as it can be observed that the estimated trend captures the decreasing trend starting from 2000, but it misses a decreasing trend also in the decade 1990-2000. Moreover, after a nadir in drinking behaviour in the 2013/14 HBSC wave, several countries/regions showed a new increase in 2017/18: Armenia (AM), Austria (AT), Flemish Belgium (BE-VLG), Bulgaria (BG), Denmark (DK), Wales (GB-WLS), Ireland (IE), Norway (NO), Sweden (SE), and Slovakia (SK) among boys and Austria (AT), Bulgaria (BG), Denmark (DK), Scotland (GB-SCT), Wales (GB-WLS), Croatia (HR), Ireland (IE), Lithuania (LT), Latvia (LV), and Sweden (SE) among girls. Countries in which the 
increase was more dramatic were Austria (AT), with +1.0 and $+7.2 \%$, among boys and girls, respectively, Bulgaria (BG), +17.3 and $+17.4 \%$, and Denmark (DK), with +4.9 and $+5.3 \%$.

\subsection{1/22. Prediction of Drinking Behavior}

Figure 1 and Figure 2 depict, for boys and girls respectively, the trends over time of weekly/daily alcohol consumption. Clusterization of trends points out differences in the observed decreasing alcohol consumption among European countries. For boys, six clusters have been detected according to the presence of ties among random effects, which occur for countries with similar trends over time, and are displayed through different colors of the panels in figures. Only Bulgaria (BG) and Hungary (HU) do not clusterize with any other country. For girls, only two clusters made up of 16 and 24 countries have been detected, meaning more similar trends among countries.

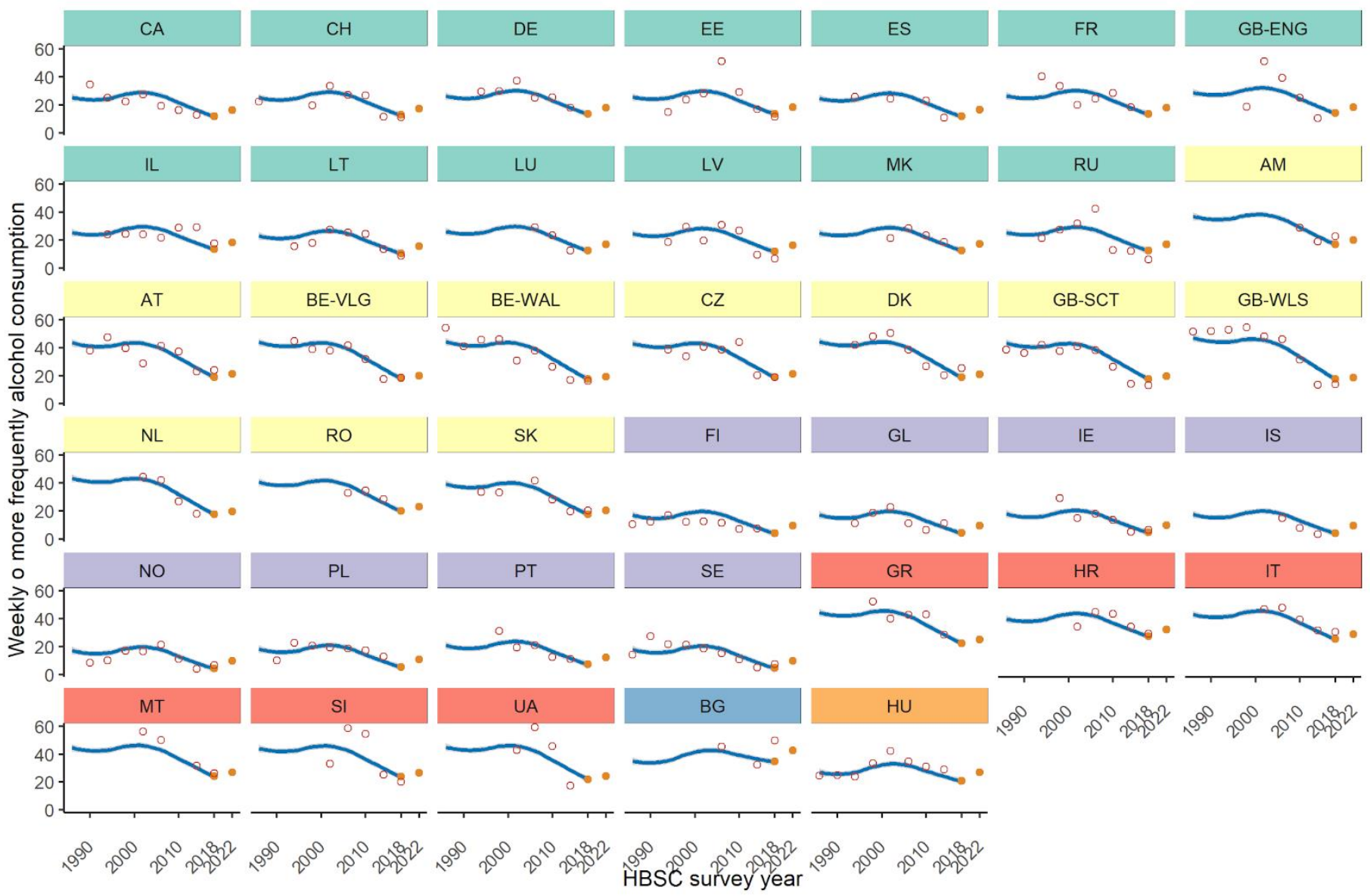

Figure 1. Trends (empty circles) and estimated (full circles for 2017/18 and 2021/22 waves) prevalences, $(\%)$, by country. Countries are grouped according to clusterization (different colors). Boys.

Table 3 shows for each country the forecast of weekly/daily alcohol consumption in the next survey wave $(2021 / 22)$ stratified by gender. The forecast is also displayed in Figures 1 and 2 (last full orange circle in each country trend).

The comparison between the predicted 2021/22 and the observed 2017/18 alcohol consumption prevalence suggests that we will expect a slight increase in the majority of the countries/regions. However, Austria (AT), Bulgaria (BG), Denmark (DK) and Italy (IT) could show a reduction in drinking behaviour in both gender groups, while in Armenia (AM) it could happen only among boys. 

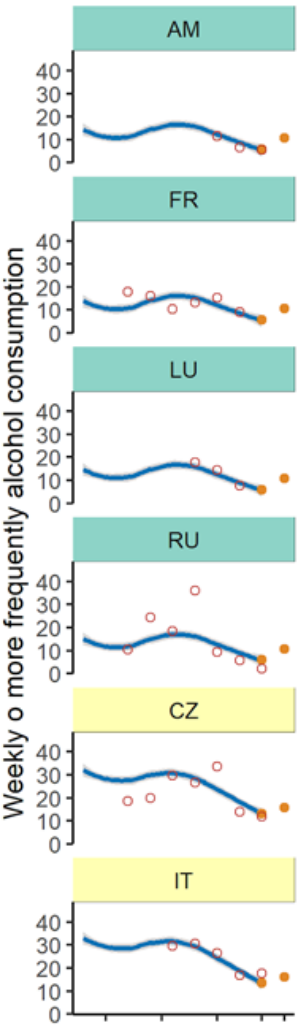

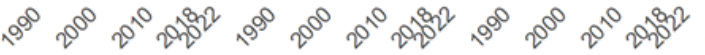
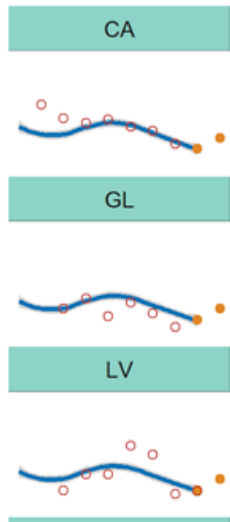

SE
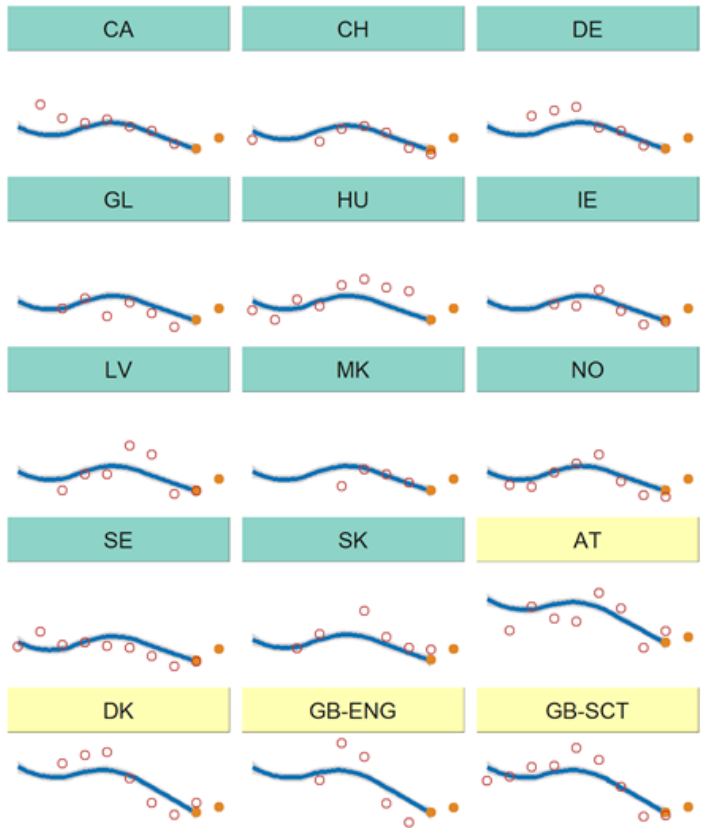

IE
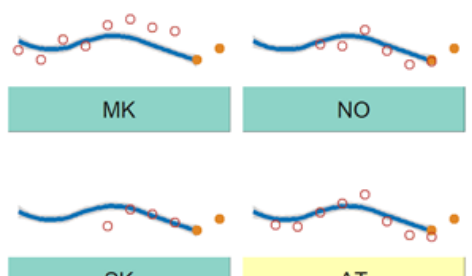

AT
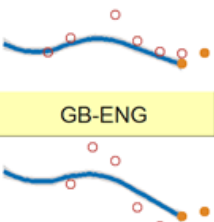

$\mathrm{NL}$
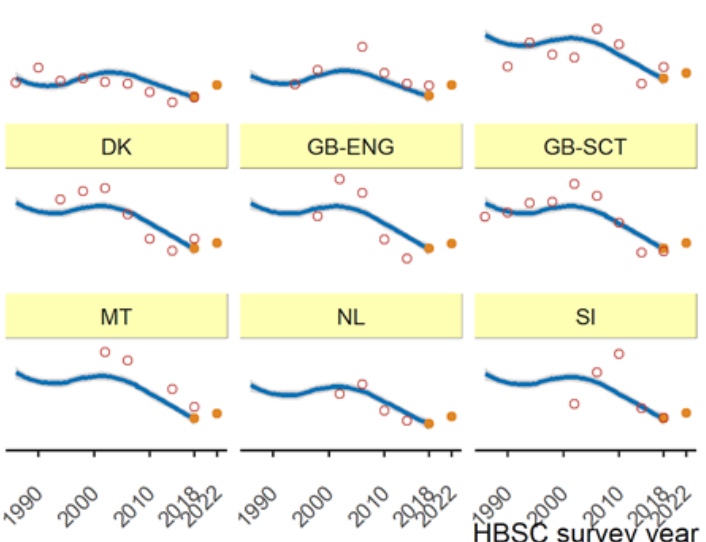

GB-SCT

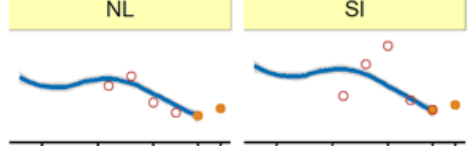

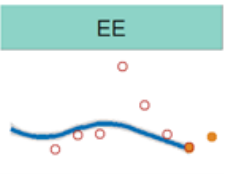

IL
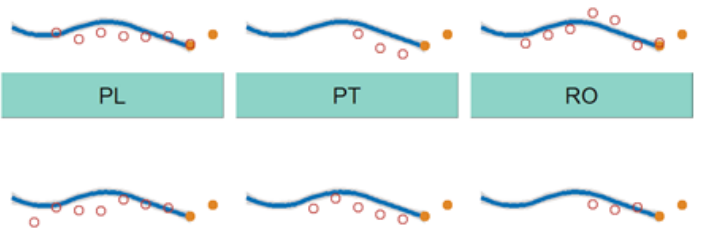

BE-VLG
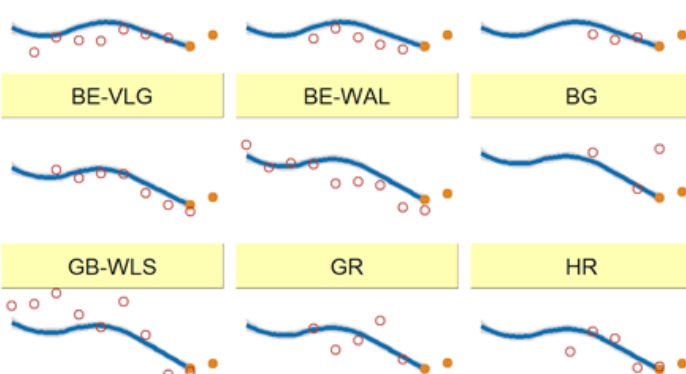

UA

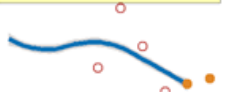

BE-WAL

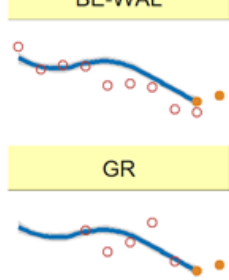

BG

HR

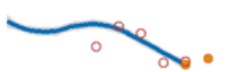

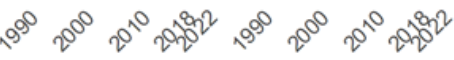

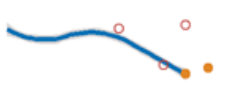

Figure 2. Trends (empty circles) and estimated (full circles for 2017/18 and 2021/22 waves) prevalences, $(\%)$, by country. Countries are grouped according to clusterization (different colors). Girls.

Table 3. 2021/22 estimated prevalence of weekly/daily alcohol consumption by country and gender.

\begin{tabular}{|c|c|c|}
\hline Country & Boys & Girls \\
\hline $\mathrm{AM}$ & 20.17 & 10.66 \\
\hline $\mathrm{AT}$ & 21.19 & 15.95 \\
\hline BE-VLG & 20.00 & 13.91 \\
\hline BE-WAL & 19.30 & 15.52 \\
\hline BG & 42.41 & 16.25 \\
\hline $\mathrm{CA}$ & 16.25 & 10.76 \\
\hline $\mathrm{CH}$ & 17.21 & 10.62 \\
\hline $\mathrm{CZ}$ & 21.21 & 15.83 \\
\hline $\mathrm{DE}$ & 17.95 & 10.77 \\
\hline DK & 20.81 & 15.89 \\
\hline $\mathrm{EE}$ & 18.23 & 10.74 \\
\hline ES & 16.34 & 12.82 \\
\hline FI & 9.57 & 10.61 \\
\hline FR & 17.79 & 10.62 \\
\hline GB-ENG & 18.10 & 15.86 \\
\hline GB-SCT & 19.52 & 15.89 \\
\hline GB-WLS & 18.50 & 15.84 \\
\hline GL & 9.64 & 10.61 \\
\hline GR & 24.99 & 16.03 \\
\hline HR & 32.24 & 15.83 \\
\hline $\mathrm{HU}$ & 27.02 & 10.66 \\
\hline IE & 9.95 & 10.62 \\
\hline IL & 18.52 & 10.62 \\
\hline IS & 9.43 & 10.60 \\
\hline IT & 28.85 & 16.04 \\
\hline
\end{tabular}


Table 3. Cont.

\begin{tabular}{ccc}
\hline Country & Boys & Girls \\
\hline LT & 15.52 & 10.62 \\
LU & 16.99 & 10.69 \\
LV & 16.45 & 10.62 \\
MK & 17.42 & 10.62 \\
MT & 26.80 & 15.93 \\
NL & 19.62 & 14.50 \\
NO & 9.67 & 10.62 \\
PL & 10.68 & 10.62 \\
PT & 12.27 & 10.62 \\
RO & 23.04 & 10.62 \\
RU & 16.99 & 10.62 \\
SE & 9.86 & 10.62 \\
SI & 26.45 & 16.10 \\
SK & 20.58 & 10.79 \\
UA & 24.15 & 15.96 \\
\hline
\end{tabular}

\subsection{Model Assessment. Comparison between 2017/18 Estimates and Observed Prevalences}

We tested the model prediction on the 23 countries for which alcohol consumption data were available in the 2017/18 survey. Tables 4 and 5 report for boys and girls, respectively, partial results from the clusterization of countries/regions and the absolute error (AE) of the model in estimating the $2017 / 18$ weekly/daily alcohol consumption by country. The $\mathrm{AE}$ of the model was computed as the difference between the estimated and observed alcohol consumption for the 23 countries that still used the question "At present, how often do you drink anything alcoholic? Try to include even those times when you only drink a small amount" in the 2017/18 HBSC wave. Mean, median, minimum and maximum errors over countries are also reported.

Table 4. Comparison between 2017/18 estimates and observed prevalence (\%) among boys.

\begin{tabular}{ccccc}
\hline Country & Observed & Predicted & AE & Cluster \\
\hline CH & 11.17 & 12.61 & 1.44 & 1 \\
EE & 11.34 & 13.56 & 2.22 & 1 \\
IL & 17.70 & 13.73 & 3.97 & 1 \\
LT & 9.01 & 10.71 & 1.71 & 1 \\
LV & 6.77 & 11.86 & 5.09 & 1 \\
RU & 6.03 & 12.52 & 6.49 & 2 \\
AM & 22.89 & 17.05 & 5.84 & 2 \\
AT & 23.86 & 18.99 & 4.87 & 2 \\
BE-VLG & 18.46 & 18.06 & 0.40 & 2 \\
BE-WAL & 16.17 & 17.57 & 1.40 & 2 \\
CZ & 18.93 & 18.97 & 0.04 & 2 \\
DK & 25.27 & 18.86 & 6.41 & 2 \\
GB-SCT & 13.22 & 17.59 & 4.37 & 2 \\
GB-WLS & 13.95 & 17.60 & 3.65 & 3 \\
SK & 20.35 & 17.79 & 2.56 & 3 \\
IE & 6.52 & 4.81 & 1.71 & 3 \\
NO & 6.85 & 4.40 & 2.45 & 4 \\
SE & 7.53 & 4.71 & 2.82 & 4 \\
HR & 29.22 & 27.54 & 1.68 & 4 \\
IT & 30.31 & 25.46 & 4.85 & 4 \\
MT & 26.13 & 24.08 & 2.05 & 5 \\
SI & 20.11 & 23.73 & 3.62 & Min-Max \\
BG & 49.70 & 34.81 & 14.89 & $0.04-14.89$ \\
Overall AE & Mean & Median & 3.05 & \\
& 3.68 & 2.82 & & 2 \\
\hline
\end{tabular}


Table 5. Comparison between 2017/18 estimates and observed prevalence (\%) among girls.

\begin{tabular}{ccccc}
\hline Country & Observed & Predicted & AE & Cluster \\
\hline AM & 5.48 & 5.80 & 0.32 & 1 \\
CH & 3.81 & 5.67 & 1.86 & 1 \\
EE & 6.31 & 5.88 & 0.43 & 1 \\
IE & 5.03 & 5.68 & 0.65 & 1 \\
IL & 6.60 & 5.67 & 0.92 & 1 \\
LT & 7.12 & 5.68 & 1.44 & 1 \\
LV & 5.43 & 5.68 & 0.25 & 1 \\
NO & 2.74 & 5.68 & 2.93 & 1 \\
RU & 2.07 & 5.89 & 3.83 & 1 \\
SE & 5.15 & 5.67 & 0.53 & 1 \\
SK & 10.39 & 5.99 & 4.40 & 2 \\
AT & 18.42 & 13.39 & 5.03 & 2 \\
BE-VLG & 7.94 & 10.69 & 2.75 & 2 \\
BE-WAL & 8.45 & 12.95 & 4.51 & 2 \\
BG & 35.09 & 13.83 & 21.26 & 2 \\
CZ & 11.86 & 13.23 & 1.37 & 2 \\
DK & 17.81 & 13.63 & 4.17 & 2 \\
GB-SCT & 12.32 & 13.62 & 1.30 & 2 \\
GB-WLS & 12.42 & 13.65 & 1.23 & 2 \\
HR & 14.31 & 13.23 & 1.08 & 2 \\
IT & 17.61 & 13.64 & 3.97 & Min-Max \\
MT & 18.70 & 13.69 & 5.01 & $0.25-21.26$ \\
SI & 14.17 & 13.70 & 0.47 & SD \\
Overall AE & Mean & Median & 4.31 & \\
& 3.03 & 1.44 & & \\
\hline
\end{tabular}

The model provided for 2017/18 forecasting an overall median error of 2.82 for boys and 1.44 for girls. The biggest error of the model (14.89 for boys and 21.26 for girls) turned out to be that for Bulgaria (BG); this country met the inclusion criteria of the study but provided data from only three non-consecutive waves $(2006,2014$ and 2018) for trends and subsequent estimates that could be an explanation of the huge AE of the model.

\section{Discussion}

The present study provided an approach to model longitudinal data, to estimate potentially nonlinear trends and forecast frequent alcohol consumption prevalence given knowledge from the past. We chose a Bayesian framework analysis due to its flexibility in relaxing assumptions on data distribution and intrinsically tying countries with similar trends. We used a data-driven dynamic clustering, which avoided us making an a priori guess on geographical patterns associated with alcohol consumption behaviours and, at the same time, borrowed strength from similar trend patterns in making estimations. That means that we nested the choice of the number of clusters inside the model and the grouping of the countries based on trend similarity. As can be further observed, the estimates of those countries with few survey waves' data were possible due to the probability of co-clustering induced by the chosen modelling approach.

\subsection{Clusters and Trends of Frequent Alcohol Consumption}

Our results appear to confirm the findings of previous research about alcohol consumption patterns within Europe. Our clusterization keeps together some historically grouped countries/regions, such as Scandinavian countries, Baltic Republics, the Czech Republic and Slovakia, and Belgian regions. However, it is difficult to find robust geographical patterns, as the data show that not all countries within the same region share similar trends. This confirms that previous classifications of drinking habits based on regional cultures (i.e., 'wet' versus 'dry' cultures) and historical traditions (i.e., Italy labelled a "wine country" or Germany a "beer country") are disappearing [21]. A homogenization 
of consumption rates and beverage preferences is increasingly evident: in recent decades, wine consumption has decreased in the traditionally 'wet' Mediterranean countries while overall alcohol consumption has increased in the northern European countries [14,22,23]. Moreover, it suggests that to explore adolescent drinking behaviour, it is necessary to take into account the role played by other factors, such as changing in social norms and ways in which young people socialize, inequalities (socioeconomic status, ethnic background), public health initiatives and policies enforced in each country (i.e., increasing taxes, age restrictions for purchasing alcohol, advertising regulations and availability restrictions) and also the strength of their implementation [24-26]. Furthermore, a recent systematic review explored the reasons for the decline of adolescent drinking and identified in the shifts in parental practices (parent-child relationship quality, parental support and involvement) the most robust evidence [27]. Finally, the indirect role of the global financial crisis of 2007-2009 should also be taken into account, and so should the COVID-19 pandemic when discussing data from the next survey.

\subsection{Comparison between 2017/18 Estimates and Observed Prevalences}

The Bayesian model we implemented has an acceptable error in forecasting alcohol consumption prevalence, as confirmed by the AE computed as the difference between the observed and the estimated prevalence in 2017/18 for the 23 countries that collected data through the same question used in the previous waves of the survey. Except for Bulgaria, with an AE of 14.89 and 21.26 for the prediction of alcohol consumption in boys and girls, respectively, only in other three countries (Russian Federation, Armenia and Denmark) is the AE greater than $5.1 \%$ for boys' weekly consumption prediction

\subsection{1/22. Forecasting of Frequent Alcohol Use}

The forecasting of weekly/daily alcohol consumption in the next wave 2021/22 shows a slight increase in most countries for both boys and girls, pointing out a potential bounce after a period of decreasing in frequent drinking habits.

The comparison between the future observed prevalence with the expected one will offer the opportunity to further investigate the potential effect of determinants occurring in the period between the two most recent waves of the HBSC survey (2018-2022). This could be even more relevant for the assessment of the pandemic effect on risk behaviours. Studies dealing with this issue showed decreased alcohol drinking among adolescents during lockdowns [28]. However, the findings of an Italian study indicate that alcoholrelated problems were even accentuated following reopening when compared with the same period in the previous year [29].

In this frame, the 2021/22 forecasts included in this study, and based on data from a thirty-year trend, could help explain any discrepancies with the prevalence of alcohol consumption observed after the pandemic.

\subsection{Strengths and Limitations}

Among the strengths of the study, the large sample and number of countries involved and the use of validated questionnaires that allow for cross-national comparisons can be accounted for. The study also has some limitations. First, the assessment of alcohol consumption is based on self-reported measures, so that alcohol consumption could be under-reported, especially among adolescents who are heavy drinkers. Second, the lack of data for some countries in specific years made it difficult to identify a robust temporal trend and to forecast the 2021/22 alcohol consumption prevalence. Moreover, the changes to the mandatory questions of the HBSC questionnaire meant that for the $2017 / 18$ survey, only just over half of the countries had data comparable to the previous waves of the surveillance. 


\section{Conclusions}

The impact of the COVID-19 pandemic and related lockdowns on adolescents' risk conducts remains an open question. We can expect that increased use of online communication with limited possibilities of personal meetings and spending spare time outside influenced drinking behaviours and accented global determinants.

From a public health perspective, monitoring (and, hopefully, forecasting) trends in adolescent alcohol use and comparing predicted and observed data can help us to make hypotheses about the effectiveness of policies and health promotion interventions, and about the reasons for any discrepancies in alcohol consumption from the expected prevalence on the basis of a thirty-year trend.

Despite almost all countries having experienced a decline in frequent alcohol consumption in the last three decades, this decrease remains largely unexplained and adolescent alcohol use a major public health concern due to both short and long-term adverse outcomes it is associated with. Unlike other substances, such as tobacco, which in recent years has shown a reverse gender gap [30,31], habitual alcohol consumption remains more prevalent among boys than girls of the same age. Nevertheless, the data confirm a narrowing of the gender gap in many countries and regions.

Finally, further research is needed to better understand the factors associated with adolescent drinking and its changes over time.

Author Contributions: Conceptualization, L.C., P.D. and P.B.; methodology, P.B., L.C., M.B., A.V., T.B. and M.Š.; formal analysis, P.B.; data curation, P.B., L.C. and M.B.; writing-original draft preparation, L.C., P.B., M.B., T.B., M.Š, A.V. and R.I.C.; writing-review and editing, P.D., P.B., L.C., M.B., A.V., R.I.C., T.B., P.N. and M.Š.; supervision, P.B., L.C. and P.D. All authors have read and agreed to the published version of the manuscript.

Funding: Italian HBSC surveys are promoted and funded by the Ministry of Health/National Centre for Disease Prevention and Control (CCM) and the Italian National Institute of Health (Project "Behavioral Risk Survey System for Ages 6-17", cap. 4393/2005-CCM,4393/2013-CCM). Slovak Research and Development Agency supports the Slovak HBSC surveys under the Contract No. APVV-18-0070. The present study was also funded by the University of Torino (CHAL_RILO_17_01 and CHAL_RILO_20_01).

Institutional Review Board Statement: The study was conducted according to the guidelines of the Declaration of Helsinki. The HBSC survey was the personal responsibility of principal investigators in each of the 40 countries and regions. For details, see http: / www.hbsc.org.

Informed Consent Statement: Passive or active consent for participation in the HBSC study was obtained by school administrators, parents or adolescents prior to participation, and in line with the regulations in each participating country. Institutional ethical approval was obtained in each participating country.

Data Availability Statement: The HBSC International Coordinating Centre is based at the University of Glasgow, UK. Data from the HBSC study can be obtained from the HBSC Data Management Centre in accordance with the HBSC data access policy. Further information on accessing HBSC data is available from: https: / www.uib.no/en/hbscdata (accessed on 30 January 2022).

Acknowledgments: The HBSC survey is a collaborative project of the WHO Regional Office for Europe. The authors would like to thank the contribution of the principal investigators and team members of each participating HBSC country. The international coordinator of the 1985/86 survey was Leif Aaro (University of Bergen, Norway); Candace Currie (Glasgow Caledonian University) coordinated the survey from the 1993/94 to the 2013/14 waves; for the 2013/14 and 2017/18 surveys, the coordinator was Joanna Inchley (University of Glasgow). For all surveys, the international Data Bank Manager was Oddrun Samdal (University of Bergen, Norway). We thank all adolescents and schools who participated in each survey and country.

Conflicts of Interest: The authors declare no conflict of interest. 


\section{Appendix A}

Table A1. Participation in HBSC waves from 1986 to 2018, by country. In the table, numbers represent how many 15-year-olds answered questions about alcohol consumption. Countries participating in at least 3 surveys (one of which in 2014 or 2018) were included in the analysis highlighted in green.

\begin{tabular}{|c|c|c|c|c|c|c|c|c|c|}
\hline Region & 1986 & 1990 & 1994 & 1998 & 2002 & 2006 & 2010 & 2014 & 2018 \\
\hline $\mathrm{AL}$ & & & & & & & & 1667 & \\
\hline $\mathrm{AM}$ & & & & & & & 156 & 105 & 204 \\
\hline AT & & 974 & 1622 & 1346 & 1223 & 1476 & 1785 & 1236 & 1254 \\
\hline $\mathrm{AZ}$ & & & & & & & & & 1274 \\
\hline BE-VLG & & & 1314 & 1512 & 1967 & 1477 & 1199 & 1680 & 1366 \\
\hline BE-WAL & 1099 & 795 & 1489 & 599 & 1349 & 1380 & 1288 & 1891 & 1596 \\
\hline BG & & & & & & 1658 & & 1619 & 1453 \\
\hline CA & & 533 & 486 & 2362 & 1169 & 2245 & 5166 & 4780 & \\
\hline $\mathrm{CH}$ & 1638 & & & 1789 & 1452 & 1455 & 2157 & 2156 & 2327 \\
\hline $\mathrm{CZ}$ & & & 342 & 1172 & 1647 & 540 & 1513 & 1737 & 3683 \\
\hline $\mathrm{DE}$ & & & 223 & 1535 & 1660 & 2492 & 1599 & 2043 & \\
\hline DK & & & 482 & 1499 & 1349 & 1448 & 1189 & 1240 & 737 \\
\hline $\mathrm{EE}$ & & & 1082 & 535 & 1267 & 692 & 853 & 735 & 1080 \\
\hline ES & & & 1458 & & 1739 & & 1972 & 3645 & \\
\hline FI & 1081 & 894 & 1132 & 1444 & 1716 & 1650 & 2077 & 1915 & \\
\hline FR & & & 1245 & 1157 & 2538 & 2168 & 1390 & 1366 & \\
\hline GB-ENG & & & & 437 & 1718 & 1430 & 1059 & 1535 & \\
\hline GB-NI & & & 1240 & 952 & & & & & \\
\hline GB-SCT & 1493 & 1138 & 1326 & 1706 & 1136 & 2053 & 2500 & 1765 & 1364 \\
\hline GB-WLS & 1975 & 1881 & 1230 & 1394 & 1144 & 1154 & 1583 & 1407 & 4117 \\
\hline GE & & & & & & & & & 170 \\
\hline GL & & & 28 & 409 & 223 & 383 & 359 & 21 & \\
\hline GR & & & & 1312 & 1301 & 1363 & 1615 & 1296 & \\
\hline HR & & & & & 1428 & 1611 & 2406 & 1845 & 1967 \\
\hline HU & 1185 & 2091 & 1735 & 732 & 1299 & 1173 & 1716 & 1084 & \\
\hline IE & & & & 1343 & 832 & 1582 & 1567 & 1411 & 1008 \\
\hline IL & & & 1309 & 1360 & 1473 & 1721 & 1315 & 1566 & 2670 \\
\hline IS & & & & & & 1837 & 3593 & 3235 & \\
\hline IT & & & & & 1211 & 1321 & 1520 & 1251 & 1278 \\
\hline $\mathrm{KZ}$ & & & & & & & & & 1483 \\
\hline $\mathrm{LT}$ & & & 1575 & 1257 & 1885 & 411 & 367 & 167 & 1134 \\
\hline LU & & & & & & 1415 & 1315 & 1030 & \\
\hline LV & & & 132 & 1176 & 1083 & 1304 & 1329 & 1693 & 1313 \\
\hline MD & & & & & & & & 1545 & \\
\hline MK & & & & & 1368 & 1880 & 1492 & 1412 & \\
\hline MT & & & & & 627 & 154 & & 185 & 156 \\
\hline NL & & & & & 1239 & 468 & 1423 & 1319 & \\
\hline $\mathrm{NO}$ & & 123 & 133 & 1551 & 1551 & 840 & 1212 & 869 & 620 \\
\hline PL & & 96 & 237 & 1609 & 2110 & 1864 & 1395 & 1466 & \\
\hline $\mathrm{PT}$ & & & & 1186 & 782 & 1332 & 1423 & 1329 & \\
\hline RO & & & & & & 1290 & 1924 & 1405 & \\
\hline RS & & & & & & & & & 1576 \\
\hline RU & & & 1354 & 1322 & 2558 & 1669 & 1757 & 1388 & 1471 \\
\hline SE & 133 & 1071 & 170 & 949 & 1195 & 1407 & 1960 & 2681 & 1494 \\
\hline SI & & & & & 1046 & 963 & 991 & 1034 & 1359 \\
\hline SK & & & 199 & 804 & & 875 & 1888 & 1797 & 1192 \\
\hline TR & & & & & & 135 & & & \\
\hline UA & & & & & 1585 & 938 & 1824 & 1628 & \\
\hline US & & & & 1753 & 1565 & 962 & 1833 & & \\
\hline
\end{tabular}

Highlight the countries that met the inclusion criteria of the study (green) and those that didn- $\mathrm{t}$ (red). 


\section{References}

1. World Health Organization. Global Status Report on Alcohol and Health 2018; World Health Organization: Geneva, Switzerland, 2018; ISBN 978-92-4-156563-9.

2. NCD Global Monitoring Framework. Available online: https://www.who.int/publications-detail-redirect/ncd-surveillanceglobal-monitoring-framework (accessed on 19 December 2021).

3. World Health Organization. Status Report on Alcohol Consumption, Harm and Policy Responses in 30 European Countries; World Health Organization: Geneva, Switzerland, 2019.

4. Charrier, L.; Canale, N.; Dalmasso, P.; Vieno, A.; Sciannameo, V.; Borraccino, A.; Lemma, P.; Ciardullo, S.; Berchialla, P.; 2018 HBSC-Italia Group; et al. Alcohol Use and Misuse: A Profile of Adolescents from 2018 Italian HBSC Data. Ann. Ist Super. Sanita 2020, 56, 531-537. [CrossRef] [PubMed]

5. Charrier, L.; Berchialla, P.; Dalmasso, P.; Borraccino, A.; Lemma, P.; Cavallo, F. Cigarette Smoking and Multiple Health Risk Behaviors: A Latent Class Regression Model to Identify a Profile of Young Adolescents. Risk Anal. 2019, 39, 1771-1782. [CrossRef]

6. Inchley, J.; World Health Organization, Regional Office for Europe. Adolescent Alcohol-Related Behaviours: Trends and Inequalities in the WHO European Region, 2002-2014; World Health Organization, Regional Office for Europe: Copenhagen, Denmark, 2018; ISBN 978-92-890-5349-5.

7. Simons-Morton, B.G.; Farhat, T.; ter Bogt, T.F.M.; Hublet, A.; Kuntsche, E.; Nic Gabhainn, S.; Godeau, E.; Kokkevi, A. HBSC Risk Behaviour Focus Group Gender Specific Trends in Alcohol Use: Cross-Cultural Comparisons from 1998 to 2006 in 24 Countries and Regions. Int. J. Public Health 2009, 54 (Suppl. 2), 199-208. [CrossRef] [PubMed]

8. Leal-López, E.; Sánchez-Queija, I.; Vieno, A.; Currie, D.; Torsheim, T.; Pavlova, D.; Moreno-Maldonado, C.; De Clercq, B.; Kalman, M.; Inchley, J. Cross-National Time Trends in Adolescent Alcohol Use from 2002 to 2014. Eur. J. Public Health 2021, 31, 859-866. [CrossRef] [PubMed]

9. Kuntsche, E.; Kuntsche, S.; Knibbe, R.; Simons-Morton, B.; Farhat, T.; Hublet, A.; Bendtsen, P.; Godeau, E.; Demetrovics, Z. Cultural and Gender Convergence in Adolescent Drunkenness: Evidence From 23 European and North American Countries. Arch. Pediatr. Adolesc. Med. 2011, 165, 152-158. [CrossRef] [PubMed]

10. Zaborskis, A.; Sumskas, L.; Maser, M.; Pudule, I. Trends in Drinking Habits among Adolescents in the Baltic Countries over the Period of Transition: HBSC Survey Results, 1993-2002. BMC Public Health 2006, 6, 67. [CrossRef]

11. Looze, M.D.; Raaijmakers, Q.; Bogt, T.; Bendtsen, P.; Farhat, T.; Ferreira, M.; Godeau, E.; Kuntsche, E.; Molcho, M.; Pfortner, T.-K.; et al. Decreases in Adolescent Weekly Alcohol Use in Europe and North America: Evidence from 28 Countries from 2002 to 2010. Eur. J. Public Health 2015, 25, 69-72. [CrossRef] [PubMed]

12. Baška, T.; Madarasová-Gecková, A.; Bašková, M.; Krajčovič, A. Decline in Alcohol Use among Adolescents in Slovakia: A Reason for Optimism? Public Health 2016, 139, 203-208. [CrossRef]

13. Vashishtha, R.; Pennay, A.; Dietze, P.; Marzan, M.B.; Room, R.; Livingston, M. Trends in Adolescent Drinking across 39 HighIncome Countries: Exploring the Timing and Magnitude of Decline. Eur. J. Public Health 2021, 31, 424-431. [CrossRef] [PubMed]

14. Leifman, H. Homogenisation in Alcohol Consumption in the European Union. Nord. Stud. Alcohol Drugs 2001, 18, 15-30. [CrossRef]

15. Kraus, L.; Seitz, N.-N.; Piontek, D.; Molinaro, S.; Siciliano, V.; Guttormsson, U.; Arpa, S.; Monshouwer, K.; Leifman, H.; Vicente, J.; et al. 'Are The Times A-Changin'? Trends in Adolescent Substance Use in Europe: Adolescent Substance Use Trends. Addiction 2018, 113, 1317-1332. [CrossRef] [PubMed]

16. Currie, C.; Griebler, R.; Inchley, J.; Theunissen, A.; Molcho, M.; Samdal, O.; Dur, W. (Eds.) Health Behaviour in School-Aged Children (HBSC) Study Protocol: Background, Methodology and Mandatory Items for the 2009/10 Survey; Child and Adolescent Health Research Unit (CAHRU): Edinburg, Scotland, 2010.

17. Roberts, C.; Freeman, J.; Samdal, O.; Schnohr, C.W.; de Looze, M.E.; Nic Gabhainn, S.; Iannotti, R.; Rasmussen, M. International HBSC Study Group The Health Behaviour in School-Aged Children (HBSC) Study: Methodological Developments and Current Tensions. Int. J. Public Health 2009, 54 (Suppl. 2), 140-150. [CrossRef] [PubMed]

18. Paddock, S.M.; Savitsky, T.D. Bayesian Hierarchical Semiparametric Modelling of Longitudinal Post-Treatment Outcomes from Open Enrolment Therapy Groups. J. R. Stat. Soc. Ser. A Stat. Soc. 2013, 176, 795-808. [CrossRef] [PubMed]

19. Escobar, M.D.; West, M. Bayesian Density Estimation and Inference Using Mixtures. J. Am. Stat. Assoc. 1995, 90, 577-588. [CrossRef]

20. R Core Team. R: A Language and Environment for Statistical Computing; R Foundation for Statistical Computing: Vienna, Austria, 2021.

21. Aresi, G.; Cleveland, M.J.; Vieno, A.; Beccaria, F.; Turrisi, R.; Marta, E. A Mixed Methods Cross-Cultural Study to Compare Youth Drinking Cultures in Italy and the USA. Appl. Psychol Health Well-Being 2020, 12, 231-255. [CrossRef]

22. Allamani, A.; Voller, F.; Kubicka, L.; Bloomfield, K. Drinking Cultures and the Position of Women in Nine European Countries. Subst. Abus. 2000, 21, 231-247. [CrossRef]

23. Gordon, R.; Heim, D.; MacAskill, S. Rethinking Drinking Cultures: A Review of Drinking Cultures and a Reconstructed Dimensional Approach. Public Health 2012, 126, 3-11. [CrossRef] [PubMed]

24. Bräker, A.B.; Soellner, R. Alcohol Drinking Cultures of European Adolescents. Eur. J. Public Health 2016, 26, 581-586. [CrossRef]

25. Brand, D.A.; Saisana, M.; Rynn, L.A.; Pennoni, F.; Lowenfels, A.B. Comparative Analysis of Alcohol Control Policies in 30 Countries. PLoS Med. 2007, 4, e151. [CrossRef] 
26. Leal-López, E.; Moreno-Maldonado, C.; Inchley, J.; Deforche, B.; Van Havere, T.; Van Damme, J.; Buijs, T.; Sánchez-Queija, I.; Currie, D.; Vieno, A.; et al. Association of Alcohol Control Policies with Adolescent Alcohol Consumption and with Social Inequality in Adolescent Alcohol Consumption: A Multilevel Study in 33 Countries and Regions. Int. J. Drug Policy 2020, 84, 102854. [CrossRef]

27. Vashishtha, R.; Livingston, M.; Pennay, A.; Dietze, P.; MacLean, S.; Holmes, J.; Herring, R.; Caluzzi, G.; Lubman, D.I. Why is adolescent drinking declining? A systematic review and narrative synthesis. Addict. Res. Theory 2019, 28, 275-288. [CrossRef]

28. Pigeaud, L.; de Veld, L.; van Hoof, J.; van der Lely, N. Acute Alcohol Intoxication in Dutch Adolescents Before, During, and After the First COVID-19 Lockdown. J. Adolesc. Health 2021, 69, 905-909. [CrossRef] [PubMed]

29. Grigoletto, V.; Cognigni, M.; Occhipinti, A.A.; Abbracciavento, G.; Carrozzi, M.; Barbi, E.; Cozzi, G. Rebound of Severe Alcoholic Intoxications in Adolescents and Young Adults After COVID-19 Lockdown. J. Adolesc. Health 2020, 67, 727-729. [CrossRef] [PubMed]

30. Gorini, G.; Gallus, S.; Carreras, G.; De Mei, B.; Masocco, M.; Faggiano, F.; Charrier, L.; Cavallo, F.; Spizzichino, L.; Galeone, D.; et al. Prevalence of Tobacco Smoking and Electronic Cigarette Use among Adolescents in Italy: Global Youth Tobacco Surveys (GYTS), 2010, 2014, 2018. Prev. Med. 2020, 131, 105903. [CrossRef] [PubMed]

31. Charrier, L.; Berchialla, P.; Galeone, D.; Spizzichino, L.; Borraccino, A.; Lemma, P.; Dalmasso, P.; Cavallo, F. Smoking Habits among Italian Adolescents: What Has Changed in the Last Decade? Biomed Res. Int. 2014, 2014, 287139. [CrossRef] 